
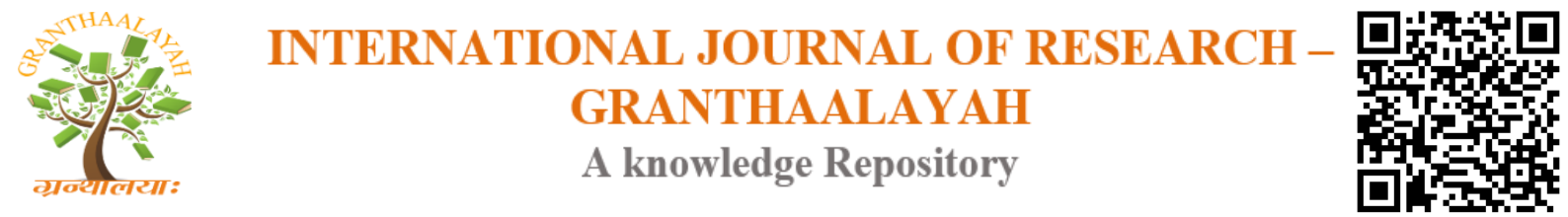

Science

\title{
DRUG ENHANCEMENT, SYNERGISM AND ANTIFUNGAL ACTIVITY OF MICONAZOLE ASSOCIATED POLYGODIAL AGAINST CANDIDA ALBICANS
}

\author{
Vagner Rodrigues Santos ${ }^{* 1}$, Livia Sâmara Franciele de Souza Pinto², Bruno César de \\ Borges Araújo e Ribeiro ${ }^{3}$, IsaoKubo ${ }^{4}$ \\ ${ }^{1}$ Associate Professor,Laboratory of Microbiology, Department of Oral Clinica, Oral Pathology, \\ Oral Surgery, Faculty of Dentistry, Universidade Federal de Minas Gerais, Belo Horizonte, \\ Brazil \\ ${ }^{2}$ Dentistry Graduation Student, PROBIC/FAPEMIG Initiation Scientific grants, Faculty of \\ Dentistry, Universidade Federal de Minas Gerais, Belo Horizonte, Brazil \\ ${ }^{3}$ Dentistry Graduation Student, Iniciation Scientific Voluntary Student \\ ${ }^{4}$ Professor of Natural Products Chemistry, Department of Environmental Science, Policy and \\ Management and Department of Nutritional Science and Toxicology, University of California at \\ Berkeley, California, USA
}

\begin{abstract}
Miconazole has low toxicity, however, there is a high incidence resistance of Candida sp. In the search for new drugs or improve existing ones, the originating products of medicinal plants has been the target of constant studies. The aim of this work was to verifyin vitrosynergism and antifungal activity of miconazole associated with poligodial. Miconazole (MCZ) and Poligodial (P) were dissolved in dimethylsulfoxide (DMSO) $1 \%$ done successive dilutions of each product ranging from $25 \mathrm{mg}$ to $0,19 \mathrm{mg} / \mathrm{mL}$ (Polygodial) $\left(10^{-1}\right.$ to $10^{-9} \mathrm{mg} / \mathrm{ml}$ ) $14 \mathrm{mg}$ to $0,10 \mathrm{mg} / \mathrm{mL}$ (miconazole). Antifungal test were based by CLSI diffusion agar against $C$. albicans (ATCC 18804), C. albicans (LMB01) and C. albicans (LMB02), C. tropicalis (ATCC 18807), C. lusitaniae (ATCC 42720), C. krusei (ATCC 20405). The results had shown all compounds that were effective in inhibiting Candida species. However, the zones of inhibition in agar diffusion test were higher for Miconazole $(16,83 \mathrm{~mm})$ and Polygodial $(15,16 \mathrm{~mm})$ while the MIX showed lower inhibition zones $(14,43 \mathrm{~mm})$ when compared with controls. MIX was more effective in MIC test, and lowest concentration were at $10^{-6}$ dilution $(0,60 \mathrm{mg} / \mathrm{ml})$ compared to $\mathrm{MCZ}$ and $\mathrm{P}$ controls.
\end{abstract}

Keywords: Candida sp; Miconazole; Polygodial; Antifungal Activity; MIC.

Cite This Article: Vagner Rodrigues Santos, Livia Sâmara Franciele de Souza Pinto, Bruno César de Borges Araújo e Ribeiro, and IsaoKubo. (2017). "DRUG ENHANCEMENT, SYNERGISM AND ANTIFUNGAL ACTIVITY OF MICONAZOLE ASSOCIATED POLYGODIAL AGAINST CANDIDA ALBICANS." International Journal of Research Granthaalayah, 5(11), 95-101. https://doi.org/10.29121/granthaalayah.v5.i11.2017.2332. 


\section{Introduction}

An increase incidence of fungal infections is associated with some predisposing factors as the use of dentures, xerostomia, prolonged therapy with antibiotics, local trauma, malnutrition, endocrine disorders, increased longevity of people, among other states that diminish the quality of individual defense [1-4]. Oral candidiasis is one of the most common clinical features of those patients infected with the human immunodeficiency virus - HIV [5, 6]. Oral candidiasis treatment is based on four fundaments: making an early and accurate diagnosis of the infection; correcting the predisposing factors or underlying diseases; evaluating the type of Candida infection; appropriate use of antifungal drugs [7-10]. Miconazole (MCZ) is a time-honored antifungal of the imidazole class. MCZ exerts a multipronged effect on fungi. It inhibits the cytochrome P450 complex. Disturbances in oxidative and peroxidative enzyme activities lead to an intracellular toxic concentration of hydrogen peroxide. As a result, intracellular organelle destruction then leads to cell necrosis [11-15].

Natural products derived from plants, have over the years greatly contributed to the development of therapeutic drugs. Polygodialis a sesquiterpene isolated from the bark of the plant Drymiswinteri (Winteraceae), Polygonumpunctatum, and other plants, that exhibit antinociceptive properties [16-18]. Polygodial exhibits several pharmacologically interesting activities. Among them, its antifungal properties have been more thoroughly studied.

Polygodial on the Saccharomycescerevisae mitochondrial energy metabolism were described and has been suggested as one of the possible targets for polygodial action inhibited the mitochondrial ATP synthesis coupled to succinate oxidation [15-22]. Polygodial showed in vitrostrong antifungal activity, comparable to amphotericin B, against Candida albicans, C. utilis, C. krusei, Cryptococcus neoformans, S. cerevisiae and also filamentous fungi including Trichophyton mentagrophytes, T. ruburum, and Penicilliummarneffei. Other strains such as Aspergillus fumigatus, A. flavus, P. chrysogenum, C. lipolytica, and C. tropicalis showed moderate to low susceptibility to polygodial[23-24].So, this papper aim to evaluate in vitrothe antifungal property of a product compound miconazole and poligodial against Candida species.

\section{Material and Methods}

\subsection{Chemicals}

Miconazole (Sigma Miconazole Nitrate Aldrich- salt- USA), Polygodial (SC 201489a; Santa Cruz Biotechnology, USA) were used in the experiment.

\subsection{Suspensions Compounds Preparation}

Each chemical was dissolved in dimethylsulfoxide at $0.1 \%$ : 14,0mg miconazole (MCZ), and $25,0 \mathrm{mg}$ Polygodial(P). For isolated tests Polygodial(P) was diluted successively $\left(10^{-1}\right.$ to $\left.10^{-7}\right)$ corresponding concentrations of $25 \mathrm{mg}$ to $0.19 \mathrm{mg}$; while Miconazole was also diluted successively $\left(10^{-1}\right.$ to $10^{-7)}$ corresponding concentrations of $14 \mathrm{mg}$ to, $0.10 \mathrm{mg}$. For tests with the MIX (MCZ + P) was associated with $3 \mathrm{ml}$ of each original suspension and became the successive dilutions to obtain the various concentrations for procedure Minimum Inhibitory Concentration (MIC). 


\subsection{Microorganisms and Antifungal Testing}

Candida albicans (ATCC 18804), Candida tropicalis (ATCC 18807), Candida lusitaniae (ATCC 42720), Candida krusei (ATCC 20405) was acquired by donation from the National Institute of Quality Control in Health (INCQS), Oswaldo Cruz Institut Foundation, Rio de Janeiro. Two $C$. albicans species (LMB01 and LMB02) were collected from the mouth of patients with candidiasis associated mucositis in Dentistry Clinical Assistance for Cancer Patient Irradiated in Head and Neck region, School of Dentistry, Federal University of Minas Gerais (FOUFMG). The two Candida samples were identified in the Laboratory of Microbiology of the School of Dentistry using the CANDIFAST® kit (International Microbio, France) and through the chromogenic medium CHOMagar ${ }^{(D i f c o, ~ U S A) . ~ T h e ~ A T C C ~ l y o p h i l i z e d ~ m i c r o o r g a n i s m s ~}$ were kept in freezer $-20^{\circ} \mathrm{C}$ until the time of inoculum preparation. The Candida albicans inoculum was made using Sabouraud dextrose broth medium (Himedia, India). An aliquot of $0.1 \mathrm{~mL}$ was left suspended overnight for 18 hours. Then $0.5 \mathrm{~mL}$ suspension containing $1,0 \times 10^{6} \mathrm{UFC} / \mathrm{ml}$ corresponding to the $0.5 \mathrm{McF}$ arland standard were plated on Sabouraud dextrose agar. For antimicrobial diffusion tests were used in accordance with Kirby-Bauer test and Clinical Laboratory Standard Institute (CLSI) [25, 26]. Discs blanc antibiogram (Laborclin, Pinhais, PR, Brazil) were soaked with $20 \mu \mathrm{L}$ of each concentration of each compound (MCZ and $\mathrm{P})$ and also the associated compounds $(\mathrm{MCZ}+\mathrm{P})$ and dispensed on agar containing Candida sp. After 24 hours at $37^{\circ} \mathrm{C}$ in bacteriological and greenhouse, readings and measurements were made the zones of inhibition observed and the minimum inhibitory concentration (MIC). The results were given considering the mean and the standard deviation in $\mathrm{mm}$ of the inhibition zones, three experiments. MIC was considered the lowest concentration that inhibited growth of the microorganism.

\subsection{Ethical Aspects}

The project study was approved by the Etic Committee of UFMG (Number CAAE0561.0.203.438-11).

\section{Statistical Analysis}

Statistical analysis was performed using the nonparametric Kruskal-Wallis test and was considered significant difference when $\mathrm{p}<0.5$.

\section{Results and Discussions}

All the samples of Candida spp tested were sensitive to antifungal. However, differences in behavior were observed. Figure 1 shows the MIC in diffusion agar test. It can be observed that the MIC for MIX compound occurred on the disk 7 , is the dilution $10^{-6}(0,65 \mathrm{mg} / \mathrm{mL})$. This value is smaller and better than was observed for miconazole and polygodial separately. In the Table 1 the antimicrobial susceptibility tests showed inhibition zones were lower for Miconazole compared with Polygodial. Moreover, the combination of the two drugs $(\mathrm{MIX}=\mathrm{MCZ}+\mathrm{P})$ showed greater sensitivity of the microorganism when compared with both antifungal alone and this showed that there was synergism between the drugs. However, it is noted that containing around the disks Miconazole there are two zones of inhibition being one as evident and 
considered as a result, and a brighter region (arrow) which can mean colonies of resistant microorganisms and also observed by Martins et al (2002). This proves the high resistance of $C$. albicans to Miconazole, which leads us to look their best performance in the control of these microorganisms. Wild Candida albicans samples (LMB01 and LMB02) were more sensitive to the three antifungals tested. However, they demonstrated greater resistance to Miconazole compared to Polygodial and MIX. C. tropicalis, C. lusitaniae e C. krusei were more sensitive to MIX and Polygodial. Dimethyl sulfoxide $0.1 \%$ used as a control did not show any inhibition signal to any of the microorganisms.

Table 1: Antifungal activity of miconazole (MCZ), poligodial (P), and $\mathrm{MCZ}+\mathrm{P}(\mathrm{MIX})$ association against Candida sp. Minimum Inhibitory Concentration $(\mathrm{MIC}=\mathrm{mg} / \mathrm{mL})$ and Means and Standard

Deviations $(\mathrm{M} \pm \mathrm{SD}=\mathrm{mm})$. Results of three experiments. *Significant difference.

\begin{tabular}{|c|c|c|c|c|c|c|}
\hline Microoganisms & \multicolumn{9}{|c|}{ Antifungals } \\
\hline & \multicolumn{2}{|c|}{ Inhibition zones $(\mathbf{m m}$ M $\mathbf{\text { DP) }}$} & \multicolumn{3}{c|}{ MIC(mg/mL) } \\
\hline & $\mathbf{M C Z}$ & $\mathbf{P}$ & $\mathbf{M I X}$ & $\mathbf{M C Z}$ & $\mathbf{P}$ & $\mathbf{M I X}$ \\
\hline C.albicans (ATCC18804) & $14,83 \pm 0,55$ & $15,16 \pm 0,50$ & $16,43 \pm 0,25^{*}$ & $0,43^{*}$ & $0,78^{*}$ & $0,65^{* *}$ \\
\hline C. albicans LMB01 & $13,00 \pm 0,00^{*}$ & $14,50 \pm 0,50$ & $17,00 \pm 0,00^{*}$ & $0,87^{*}$ & 0,78 & $0,32^{* *}$ \\
\hline C. albicans LMB02 & $12,75 \pm 0,25^{*}$ & $14,00 \pm 0,00$ & $16,85 \pm 0,75^{*}$ & $0,87^{*}$ & $0,39^{*}$ & $0,65^{* *}$ \\
\hline C. tropicalis (ATCC18807) & $13,00 \pm 0,00$ & $14,36 \pm 0,76^{*}$ & $17,00 \pm 0,00^{*}$ & $0,21^{*}$ & 0,39 & $0,32^{* *}$ \\
\hline C. lusitaniae (ATCC42720) & $15,00 \pm 0,00$ & $14,60 \pm 0,50^{*}$ & $18,50 \pm 0,34^{*}$ & 0,87 & 0,78 & $0,65^{* *}$ \\
\hline C. krusei (ATCC20405) & $11,00 \pm 0,00^{*}$ & $15,75 \pm 0,45$ & $18,00 \pm 0,00^{*}$ & $1,75^{*}$ & $0,39^{* *}$ & 0,65 \\
\hline
\end{tabular}

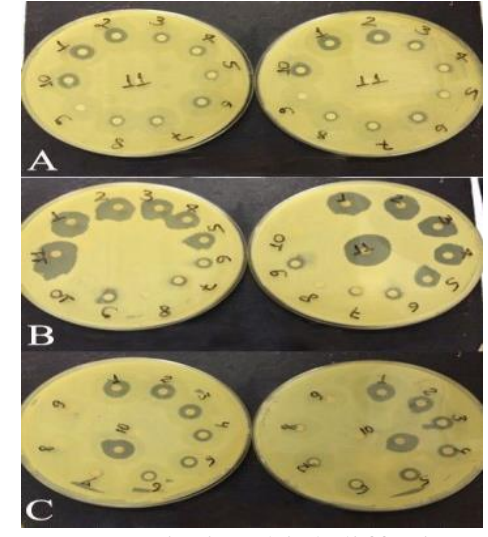

Figure 1: Antimicrobial diffusion agar test (MIC) against Candida albicans. Inhibition zones $(\mathrm{A}=$ Miconazole; $\mathrm{B}=$ Polygodial; $\mathrm{C}=\mathrm{MIX})$.

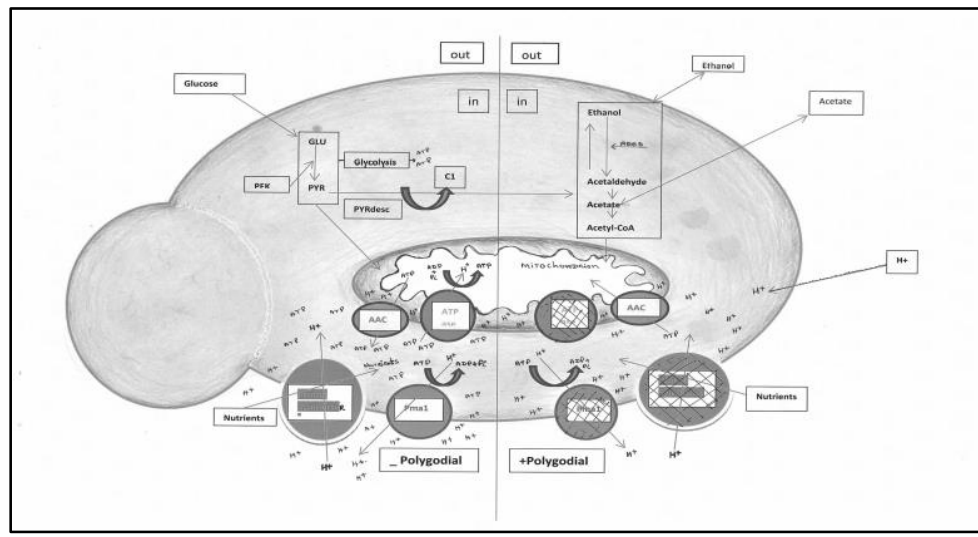

Figure 2: Polygodial activity mechanism of action in OUT and IN mitochondria yeast cell. (Adapted from Linde and Kubo, 2001; Kubo and Himejima, 1992) ${ }^{20-23}$

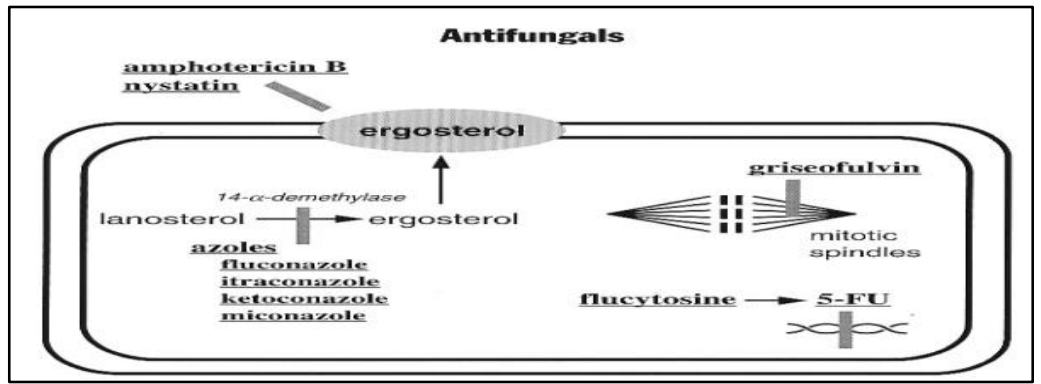

Figure 3: Mechanism of action involving the inhibition of miconazole synthesis of ergosterol (MCZ) of class Imidazole. MCZ inhibits the cytochrome P450 complex, including the enzyme

$14 \alpha$-demethylase required for ergosterol biosynthesis, in fungal cell membranes(39) 


\section{Discussion}

The choice of two wild $C$. albicans species, collected from the mouth of patients with candidiasis and irradiated in the head and neck has allowed us to observe the behavior of the standard ATCC species comparing it to generally resistant samples by exposure to chemotherapy and radiation and, also, because the vast majority of irradiated patients generally had contact with some antifungal during treatment. Wild specimens of Candida spp showed increased resistance to Miconazole. This was probably due to prior contact of patients with some of the antifungal azoles group due to treatment of candidiasis. This resistance can also be caused by radiation and chemotherapy, despite not having a concrete base on this fact.

This study suggests that the mixture compound MIX has a very good profile of antifungal activity in terms of effectiveness and spectrum of action while being devoid of any significant toxicity.The antifungal activity of two drimanesesquiterpenedialdehydes, polygodial and warburganal, alone and in combination with several other substances, was examined against three fungi, Candida albicans, Saccharomyces cerevisiae and Pityrosporumovaleemploying a broth dilution method. Anethole significantly synergized the activity of the two sesquiterpenoids against $C$. albicans and $S$. cerevisiae; however, it had only an additive effect against $P$. ovale[27].

Miconazole (MCZ) inhibits the cytochrome P450 complex, including the $14 \alpha$-demethylase enzyme required for ergosterol biosynthesis, in fungal cell membranes. Disturbances in oxidative and peroxidative enzyme activities lead to an intracellular toxic concentration of hydrogen peroxide. As a result, intracellular organelle destruction then leads to cell necrosis [12].

These biological activities add up and act synergistically enhancing the antimicrobial activity of MIX $(\mathrm{MCZ}+\mathrm{P})$. Poligodial acts as a surfactant and this feature makes it easy to miconazole activity. The poligodialmechanism of action against $S$. cerevisiae were proposed by Lunde and Kubo [28].

The fungicidal action of the primary Poligodial comes in part from its ability to function as a nonionic surfactant, disrupting the protein-lipid interface and integral proteins denaturing its conformation. As a result, the antifungal dialdehydesesquiterpene this mechanism is associated with the functions of the membrane or membrane disarray. For example, the process of acidification of the medium induced by glucose Saccharomyces cerevisiae was inhibited by Poligodial presumably caused by inhibition of $\mathrm{H}+$ in the plasma membrane ATPase by. Thus, the potent antifungal activity of Poligodial holds in its multiple functions [23].

As resistance to azoles (the preferred treatment) is occurring, we aimed at identifying compounds that increase the activity of miconazole against Candida albicans biofilms [34]. Enhancement of the antibiofilmactivity of miconazole by artesunate, pointing to potential combination therapy consisting of miconazole and artesunate to treat $C$. albicansbiofilm-related infections [29].Combinations of MUC7 12-mer and Hsn5 12-mer, and of MUC7 12-mer and miconazole have a synergic antifungal effect on $C$. neoformansandC. albicans[30-33]. 
There was a significant difference between the zones of inhibition when compared miconazole, Polygodial and MIX. These differences may be related to the types of molecules of each compound, whereas polygodial displays a heavier molecule that miconazole this contributes to the diffusion in agar is smaller [34]. This difference was also observed in the MIX and probably the polygodial interfere with the spread of miconazole. Moreover, the association of the two compounds improved antifungal activity as shown in Table 1 , for the MIC was $0,65 \mathrm{mg} / \mathrm{ml}$, the observed difference was significant when compared separately. The polygodial surfactant activity improves the action of miconazole and Candida is attacked on two fronts: in the cell wall by the absence of ergosterol and fall of the energy produced by mitochondria and disruption of intracytoplasmic membrane system [20,35].The search for novel antifungal agents and the attempt to modify and improve the features of the currently available chemotherapeutic agents are in high demand as a consequence of the rapid spread of multidrug-resistant fungal pathogens. In this regard, naturally occur- ring antimicrobial peptides are an attractive option, what is discussed by others [36-38]. In conclusion, these results suggest that the combination of miconazole with polygodial may be a promising avenue in the search for new antifungal formulations for treatment of oral candidiasis.

Conflict of Interest: The authors declare no conflicts of interest related to this research.

Acknowledgements: The authors thank FAPEMIG for financial support and fellowship of scientific initiation. Also Silvana Maria de Souza for technical support.

\section{References}

[1] Singh A., R. Verna and A. Agrawal, J. Oral. Maxillofac. Pathol.18, 2014, S81 - 85.

[2] Collins C.D., S. Cookinham and J. Smith, Patient Preferency Adherence. 5, 2011, 369-374.

[3] Hebecker B., Naglik JR, Hube B and Jacobsen ID. Expert. Review Anti-Infectuous. Therapy.12, 2014, 867-879.

[4] Noronha V.R.A.S., G.S. Araujo, R.T. Gomes, S.H. Iwanaga, M.C. Barbosa, E.N. Abdo, E. Ferreira e Ferreira, A.C. V. Campos, A.A. Souza, S.R. Abreuand V.R. Santos, Current Clinical Pharmacology. 9, 2014, 359 -364.

[5] Gaitán-Cepeda L.A., O. Sánchez-Vargas and N. Castillo, International Journal STD AIDS. 26, 2015, 625-632.

[6] Owotade F.J, M. Patel. Oral Surgery Oral Medicine Oral Pathology Oral Radiology. 118, 2014, 455- 460.

[7] Coronado-Castellote L., and Y. Jiménez-Soriano. Journal Clinical Experimental Dentistry.5, 2013, e279 -e286.

[8] Costa E., S. Silva, F. Tavaria, M. Pintado. Pathogens. 3, 2014, 908 -919.

[9] Gebremedhin S., B. Dorocka-Bobkowska, M. Prylinski, K. Konopka, and N. Duzgunes. Journal of Physiology and Pharmacology. 65, 2014, 593-600.

[10] Hu Z., J. Zhang, and X. Cheng. Pharmacology Biology. 53, 2015, 251.

[11] MetugriachukY., O. Kuroi, K. Pavasuthipaisit, J. Tsuchiya, E. Minelli, R. Okura, E. Fesce, and F. Marotta. Chinese Journal of Digestive Diseases. 6, 2005, 98 -103.

[12] PiérardG., T. Hermanns-Lê, Delvenne P, and C. Piérard-Franchimont, Expert Opinion Pharmacotherapy. 13, 2012, $1187-1194$.

[13] Wong-Paz J.E., J.C. Contreras-Esquivel, R. Rodríguez-Herrera, M.L. Carrillo-Inungaray, L.I. López, G.V. Nevárez-Moorillón, and C.N. Aguilar. Asian Pacific Journal of Tropical Medicine.8, 2015, $104-111$. 
[14] Lalla R.V., L.L. Patton, and A. Dongari-Bagtzoglou. Journal of Californian Dental Association. 41, 2013, 263-268.

[15] Garcia-Cuesta C., M.G. Sarrion-Pérez, and J.V. Bagán. Journal of Clinical Experimental Dentistry. 6, 2014, 576-582.

[16] Almeida T., F. Ribeiro, H. Kloos, and C. Zani. Memoria do Instituto Oswaldo Cruz. 96, 2001, 831-833.

[17] Malheiros A., V. CechinelFilho, C.B. Schmitt, R.A. Yunes, A. Escalante, L. Svetaz, S. Zacchino, and F. DelleMonache. Journal of Pharmacy and Pharmaceutical Sciences. 8, 2005, 335 - 339.

[18] Mecchi M.C. and J.H. Lago. Nat Prod Res. 27, 2013, 1927 -1929.

[19] Derita M., I. Montenegro, F. Garibotto, R.D. Enriz, M.C. Fritis, and S.A. Zacchino. Molecules. 18, 2013, 2029-2051.

[20] Kubo I., K. Fujita, and S.H. Lee. Journal of Agricultural Food and Chemistry. 49, 2001, 16071611.

[21] Kubo I., K. Fujita, S.H. Lee, and T.J. Ha. Phytotherapy Research. 19, 2005, 1013- 1017.

[22] McCallion R.F., A.L. Cole, J.R. Walker, J.W. Blunt and M.H. Munro, Planta Medica.44, 1982, 134-138.

[23] Leonard CM. and AM Viljoen. Journal of Ethnopharmacolology. 165, 2015, 260-285- .

[24] Lee S., J.R. Lee, C. Lunde, and I. Kubo. Planta Medica. 65, 21999, 204-208.

[25] Fujita K. and I. Kubo. Bioorganic Medicinal Chemistry. 13, 2005, 6742 -6747.

[26] De Luca M.P., J.R. Franca, F.A. Macedo, L. Grenho, M.E. Cortes, A.A. Faraco, A.N. Moreira, and V.R. Santos. Biomedicine Research International. 2014, <https://www.hindawi.com/journals/bmri/2014/348647/>doi: 10.1155/2014/348647,08 p.

[27] Franca J.R., M.P. De Luca, T.G. Ribeiro, R.O. Castilho, A.N. Moreira, V.R. Santos, and A.A. Faraco. BMC Complementary and Alternative Medicine. 14, 2014, 478, 11 p.

[28] Clinical and Laboratory Standards Institute. Performance standards for antimicrobial susceptibility testing. In twentieth informational supplement, CLSI document M 100-S20 (Wayne, PA, USA 2010), 238p..

[29] Lunde C.S., and I. Kubo. Antimicrobial Agents Chemotherapy. 44, 2000, 1943-1953.

[30] De Cremer K., E. Lanckacker, T.L. Cools, M. Bax, K. de Brucker, P. Cos, B.P. Cammue, and K. Thevissen. Antimicrobial Agents Chemotherapy. 59, 2015, 421-426.

[31] Wei G.X. and L.A Bobek. Journal of Antimicrobial Chemotherapy. 53, 2004, 750-758.

[32] Sheikh S., D. Gupta, S. Pallagatti, I. Singla, R. Gupta, and V. Goel. New York State Dental Journal. 79, 2013, 58-64.

[33] Scodavolpe S., S. Quaranta, B. Lacarelle, and C. Solas. Annals of Biology and Clinics 72, 2014, 391-404.

[34] Koletar S.L., in Diagnostic Microbiology, edited by C.R.Mahon, G. Manuselis Jr Saunders, Phyladelfia, 1995.

[35] Tian J., X. Ban, H. Zeng, J. He, Y. Chen, and Y. Wang. PLoS One. 7, 2012, e30147, 10p..

[36] Gordaliza M.. Mar Drugs. 10, 2012, 358 -402.

[37] Campbell B.C., K.L. Chan, and J.H. Kim. Frontiers of Microbiology. 3, 2012, 79.

[38] MoyeenulHuq A. K. M., J.A. Jamal, and J. Stanslas. Evidence Based Complementary and Alternative Medicine. 2014, 11 p. https://www.hindawi.com/journals/ecam/2014/782830/>doi: 10.1155/2014/782830.

[39] Figure 3. http://pharmacologycorner.com/study-aids-for-antifungals-pharmacology-diagramshowing-moa-and-table-summary-for-activity-spectrum/, November 12 2017)

*Corresponding author.

E-mail address: vegneer2003@yahoo.com.br 